\title{
Running head: LYING RELIES ON THE TRUTH
}

\author{
Lying relies on the truth \\ Evelyne Debey ${ }^{1}$, Jan De Houwer ${ }^{1}$, \& Bruno Verschuere ${ }^{1,2,3}$
}

1. Faculty of Psychology and Educational Sciences, Department of ExperimentalClinical and Health Psychology, Ghent University, Ghent, Belgium

2. Faculty of Social and Behavioural Sciences, Department of Clinical Psychology, University of Amsterdam, Amsterdam, The Netherlands

3. Faculty of Psychology and Neuroscience, Maastricht University, Maastricht, The Netherlands

Correspondence concerning this article should be addressed to Evelyne Debey, Department of Experimental Clinical and Health Psychology, Ghent University, Henri Dunantlaan 2, 9000 Ghent, Belgium. E-mail: evelyne.debey@ugent.be. Tel.: +32 (0)9 26494 46. Evelyne Debey is supported by Ghent University Grant BOF01D01010. Jan De Houwer is supported by Ghent University Grant BOF09/01M00209. 


\begin{abstract}
Cognitive models of deception focus on the conflict-inducing nature of the truth activation during lying. Here we tested the counterintuitive hypothesis that the truth can also serve a functional role in the act of lying. More specifically, we examined whether the construction of a lie can involve a two-step process, where the first step entails activating the truth, based upon which a lie response can be formulated in a second step. To investigate this hypothesis, we tried to capture the covert truth activation in a reaction-time based deception paradigm. Together with each question, we presented either the truth or lie response as distractors. If lying depends on the covert activation of the truth, deceptive responses would thus be facilitated by truth distractors relative to lie distractors. Our results indeed revealed such a "covert congruency" effect, both in errors and reaction times (Experiment 1). Moreover, stimulating participants to use the distractor information by increasing the proportion of truth distractor trials enlarged the "covert congruency" effects, and as such confirmed that the effects operate at a covert response level (Experiment 2). Our findings lend support to the idea that lying relies on a first step of truth telling, and call for a shift in theoretical thinking that highlights both the functional and interfering properties of the truth activation in the lying process.
\end{abstract}

Key words: deception, cognitive processes, truth activation, distractors 
Lying relies on the truth

\section{Introduction}

A growing body of evidence supports the long held assumption that lying is more cognitively demanding than truth telling (Vrij, Fisher, Mann, \& Leal, 2006; Zuckerman, DePaulo, \& Rosenthal, 1981). Behavioral research, for example, has shown that the additional cognitive processes involved in lying are reflected in longer latencies and a higher error rate compared to truth telling (see Verschuere \& De Houwer, 2011, for a review). Brain imaging studies have also demonstrated that, in contrast to truth telling, the act of lying involves more activity in prefrontal brain regions (anterior cingulate, dorsolateral prefrontal, and inferior frontal regions) that are known to be crucially involved in cognitive control (see Abe, 2011; Christ, Van Essen, Watson, Brubaker, \& McDermott, 2009, for reviews). The idea that cognitive control plays a key role in deception is further bolstered by studies showing that the ability to deceive is poor in children, whose prefrontal cortex development is not yet complete (Hala \& Russell, 2001; Talwar \& Lee, 2008), and in patients with neurodevelopmental and neurodegenerative conditions of the frontal lobe, such as autism and Parkinson's disease (Abe, 2009).

Despite the increasing interest in the cognitive aspect of deception, research that has attempted to unravel the underlying mechanisms as to why lying is more mentally challenging, remains relatively limited. So far, most research favors the explanation that cognitive control would be needed to resolve the response conflict evoked by the automatic activation of the dominant truth response. Self-reports, for example, have revealed that the truth often first enters ones working memory before responding deceptively (Walczyk, Roper, Seemann, \& Humphrey, 2003). Furthermore, electroencephalography (EEG) and brain imaging studies have pointed to the existence of response conflict and inhibition during deception (e.g., Christ et al., 2009; Johnson, Barnhardt, \& Zhu, 2004). Other studies provided 
evidence for the initial truth activation when lying by tracking arm movements (Duran, Dale, \& McNamara, 2010) or applying electromyography (EMG; Hadar, Makris, \& Yarrow, 2012; Seymour \& Schumacher, 2009) during a manual deception task. In the study of Duran and colleagues (2010), participants answered autobiographical yes/no questions truthfully or deceptively by moving a Nintendo Wii remote to a "yes" or "no" response label in each corner of a large screen. An analysis of the arm trajectories revealed that during deception there was a strong attraction toward the truthful response. Using a Concealed Information Test (Lykken, 1959; Verschuere, Ben-Shakhar, \& Meijer, 2011), Seymour and Schumacher (2009) asked participants to categorize previously presented words and new words as "old" or "new" by making a left or right arm movement. To some of the previously presented words, participants were instructed to deceptively indicate that they were new. The crucial measure was the proportion of partial-error responses, that is, responses that showed EMG activity in the arm associated with the incorrect response prior to EMG activity in the arm associated with the correct response. The higher proportion of partial-error responses during deceptive responding versus truthful responding revealed initial truth activation during deception. Finally, in the study of Hadar and colleagues (2012), participants used their finger or thumb to respond either truthfully or deceptively with regard to facial familiarity. EMG activity, induced by transcranial magnetic stimulation (TMS) on the primary motor cortex (i.e., motorevoked-potentials, MEPs), served as an index of the action tendency strength. The results showed that during the preparation of a lie, the MEP of the digit associated with truthful responding became larger than the MEP of the digit linked to the deceptive response. In sum, the above studies lend support for the idea that the truth is covertly activated when lying. The truth is thereby mainly depicted as causing conflict.

Also contemporary cognitive models of deception have highlighted the conflictinducing character of the truth. The Activation-Decision-Construction-Action Theory 
(Walczyk, Harris, Duck, \& Mulay, 2014; see Walczyk, Mahoney, Doverspike, \& GriffithRoss, 2009; Walczyk et al., 2003, for previous versions of the model), for instance, holds that upon hearing a question, relevant information from long-term memory will be activated and transferred into working memory. Among the relevant information, the truth would receive the highest activation, and most often be automatically activated. Once having decided to lie, the model assumes that inhibition is needed to prevent the truth from slipping out. Also the Sheffield model (Spence et al., 2004) and the Working Model of Deception (Vendemia, Buzan, \& Simon-Dack, 2005; see Vendemia, Schillaci, Buzan, Green, \& Meek, 2009, for an update) hold that generating a deceptive response requires the inhibition of the prepotent truth response. Finally, the Parallel Task-Set model (Seymour, 2001) serves to explain the RT increase for deceptive responses in the Concealed Information Test. The model departs from a dual-process account and states that items will automatically trigger two task-sets that run in parallel. Whereas one task-set would initiate a fast, automatic response based on familiarity, the other task-set would instigate a slower, more controlled correct response based on recollection. On truth trials the task-sets match, and the fast familiarity-based response can be executed. On deception trials the familiarity-based "old" response needs to be aborted in favor of the slower recollection-based "new" response. Again, the truth is argued to conflict with the selection of the lie response.

Yet, can the truth also play a more positive role in lying? A first hint in this direction comes from descriptions on the role of the executive functions of working memory updating and shifting in lying. Several authors imply that in order to produce a deceptive response, liars would need to keep the truth active in working memory, and be able to shift between the mental sets associated with these responses (Christ et al., 2009; Evans \& Lee, 2013; VisuPetra, Varga, Miclea, \& Visu-Petra, 2013). However, why exactly the truth would be kept active and be part of a shifting process during the construction of lies was left unspecified. A 
second indication comes from a study of Walczyk et al. (2003) in which many participants reported using the truth in order to lie. In the case of open-ended questions, $70 \%$ of the participants indicated that the truth was used to cue information with which to create the lie. When asked yes/no questions, $76 \%$ of the participants stated that they applied the simple rule of saying the opposite of the truth. Third, the closer liars stick to the truth, the harder it is to detect deception (Hershkowitz, 2001; Vrij, Granhag, \& Mann, 2010). Fourth, the models described above allow room for our counterintuitive idea that the truth can be functional in the lying process. The Activation-Decision-Construction-Action Theory, for example, argues that the truth prompts a network of semantic and episodic nodes in long-term memory, which can be accessed to construct a lie (Walczyk et al., 2014). Furthermore, the Working Model of Deception states that lying involves the process required for truth telling (Vendemia, Buzan, \& Simon-Dack, 2005). These considerations point to the possibility that the truth may initially be functional in constructing the lie via a two-step process, in which in a first step the truth is activated, based upon which an alternative (i.e., deceptive) response can be generated in a second step. ${ }^{1}$ Such a hypothesis not only provides new insight into the nature of lying, but can also explain why lying is more cognitively demanding than telling the truth: Lying involves truth telling plus a second process. Lying is thus cognitively more demanding than truth telling to the extent that the second process requires cognitive resources.

Until now, the evidence for a two-step process is limited. Given that the validity of subjective verbal reports about mental processes can be questioned (e.g., Nisbett \& Wilson, 1977; Pronin, 2009; Wilson, 2002), the introspective reports in the study of Walczyk et al. (2003) may only have reflected what people think about how they generate lies, not how they actually generate lies. Therefore, we report two experiments in which we used a reaction-time paradigm to obtain direct support for a two-step process in the act of lying, in which the lie response is created via a first step of truth activation. The basic logic behind the task was that 
if lying depends on a first step of truth telling, then stimuli that help or hinder truth telling might have the same effect on lying.

In our experiments, participants performed the Sheffield lie test (Spence et al., 2001), a deception paradigm in which participants answer simple yes/no questions (e.g., "Are you in Belgium?") as quickly and accurately as possible by pressing a "yes" or "no" response key. Critically, a cue instructs participants to lie or tell the truth. For instance, the color of the response labels may be used to signal lying versus truth telling. Typically, reliable lie effects in reaction times and errors are obtained in this paradigm (i.e., longer reaction times and more errors on lie trials than on truth trials; e.g., Spence \& Kaylor-Hughes, 2008; Verschuere, Spruyt, Meijer, \& Otgaar, 2011).

To investigate the two-step hypothesis of lying, we applied a manipulation that was inspired by a paper of Seymour (1977), in which the processes underlying Stroop-like congruency effects were examined. In his first experiment, colored season names served as stimuli. The ink colors used were those that are typically associated with the seasons (i.e., green for spring, yellow for summer, brown for autumn, and white for winter). One group of participants was instructed to name the season opposite to the one related to the printed ink color. When, for instance, the season name was printed in yellow, the season opposite to summer (i.e., winter) had to be said out loud. However, because word reading is more dominant than color naming (MacLeod, 1991), the presented task-irrelevant season names were expected to affect responding. Surprisingly, facilitation occurred when the season matched the printed ink color and therefore differed from the to-be-emitted response. For example, it was easier to respond "winter" when "SUMMER" was presented in yellow, than when "WINTER" was presented in yellow. This finding is suggestive for a two-step process underlying opposite season naming, where first the season associated with the ink color is covertly activated (e.g., summer when the color is yellow), based upon which the opposite 
season is then formulated (i.e., winter). When the presented season and color match, the season is congruent with the covert response of the first step. As a result, the opposite season can be easily deduced in a second step. When conversely, the color and season do not match, the season is incongruent with the required covert response. This gives rise to a covert conflict at the first stage that leads to a delay in overt responding.

If lying in our Sheffield lie test first requires the covert activation of the truth, a similar scenario as in the opposite naming task was expected to unfold. More specifically, we anticipated lying to be easier in a context that should facilitate the covert truth activation, compared to a context that should interfere with this activation. To test this prediction, we presented distractors that flanked the questions in the Sheffield lie test. The distractors represented either the truth or lie response to these questions (or were neutral symbols). Even though the distractors were task-irrelevant, we expected that they would bias responding because they overlapped with the task-relevant responses (Kornblum, Hasbroucq, \& Osman, 1990). Based on the assumption that lie construction may involve a two-step process, we expected to observe an effect of covert congruency in reaction times and errors. Truth distractors would be congruent with the covert truth response that is required in the first step of lie construction, whereas lie distractors would be incongruent with the truth response. Hence, relative to lie distractors, truth distractors should facilitate truth activation. If lying depends on this initial truth activation, then also lying should therefore be facilitated with truth distractors relative to lie distractors. In sum, according to the two-step assumption, lying should paradoxically be faster and more accurate in the presence of truth distractors than in the presence of lie distractors. 


\section{Experiment 1}

\subsection{Method}

2.1.1. Participants. Twenty-six undergraduate students at Ghent University ( 2 men, $M_{\text {age }}=20.85$ years, $\left.S D=1.83\right)$ participated in exchange for $€ 4$. All participants had normal or corrected-to-normal vision.

2.1.2. Apparatus. The Sheffield lie test was presented on a Pentium IV personal computer with a 17-inch color monitor running Tscope (Stevens, Lammertyn, Verbruggen, \& Vandierendonck, 2006). Participants sat approximately 50 centimeters from the computer screen.

\subsection{Procedure}

The experiment was run in a dimly lit room. After giving informed consent, participants were asked to fill out a questionnaire that consisted of the 20 Dutch yes/no questions that would be used in the Sheffield lie test (see Appendix A). The questions started with "Are you (a)". Half of the questions could be answered by a truthful "yes" response (e.g., "Are you a student?"), whereas the other half required a truthful "no" response (e.g., "Are you outside?"). The response options were yes, no, or unsure. In case of an "unsure" response $(<1 \%)$ or an unexpected response (e.g., "no" to "Are you an adult?"; $<1 \%)$, the experimenter discussed with the participants their response choice. This helped participants to choose or change the truthful response they would use in the Sheffield lie test. The behavioral responses to these questions in the Sheffield lie test were nevertheless excluded from further analyses (1.92\% data loss).

The instructions of the Sheffield lie test stressed both speed and accuracy. Participants were told that the distractors should be ignored. Nevertheless, we presented the distractors in such a way that they were difficult to ignore. More specifically, we divided the questions into 
two parts. The fixed part "Are you (a)" was presented in white on top of a black screen and remained there throughout the test (see Figure 1; Note that we provide the English translation here, whereas we used the Dutch phrasing, i.e., "Ben je (een)", with the "JA" and "NEEN" response labels). Also the response labels "YES" and "NO" were consistently presented according to the response mapping (counterbalanced across participants) in white on the bottom of the screen. Each trial started with a $1 \mathrm{~s}$ presentation of these fixed features. Next, a fixation cross appeared that remained in the middle of the screen for $200 \mathrm{~ms}$. Following another fixed features screen that lasted $300 \mathrm{~ms}$, the crucial word of the question was presented (Arial, 34). To avoid that participants could ignore the distractors by focusing on a fixed position, the target words were presented randomly on one of four vertically centered positions on the screen $(-5,-2,2$, or $5 \mathrm{~cm}$ from the middle of the screen). Below and above the crucial word, identical distractors were presented (Arial, 34). The vertical distance between the center of the crucial word and the center of the distractors was $1.0 \mathrm{~cm}$, subtending 1.15 visual angle from a viewing distance of $50 \mathrm{~cm}$. The distractors could be the words "YES", "NO", or the neutral hatch signs “\#\#”or “\#\#\#”. Depending on the question, the "YES" and "NO" distractors represented the truth or lie response. To further enhance processing of the distractors, we colored the target words instead of the response labels to instruct participants about whether to lie or tell the truth. According to the color rule, which was counterbalanced across participants, one color (e.g., blue) indicated to lie, whereas the other color (e.g., yellow) designated to tell the truth. Distractors were presented in a white color. Participants responded "yes" or "no" by pressing the "4" or "6" key on the numeric pad of an AZERTY keyboard. After a response, or if no response was given within $3 \mathrm{~s}$ after the word presentation, the next trial was presented. At the start of the test, participants performed two practice phases: In the first phase, 12 trials were presented without distractors; in the second phase, participants performed 24 trials in which distractors were presented. Next, 480 test trials were 
presented that were generated according to a 20 (Question) x 2 (Task [Truth vs. Lie]) x 3

(Distractor [Neutral distractor, Truth distractor, Lie distractor]) design. A self-paced break was provided in the middle of the task.

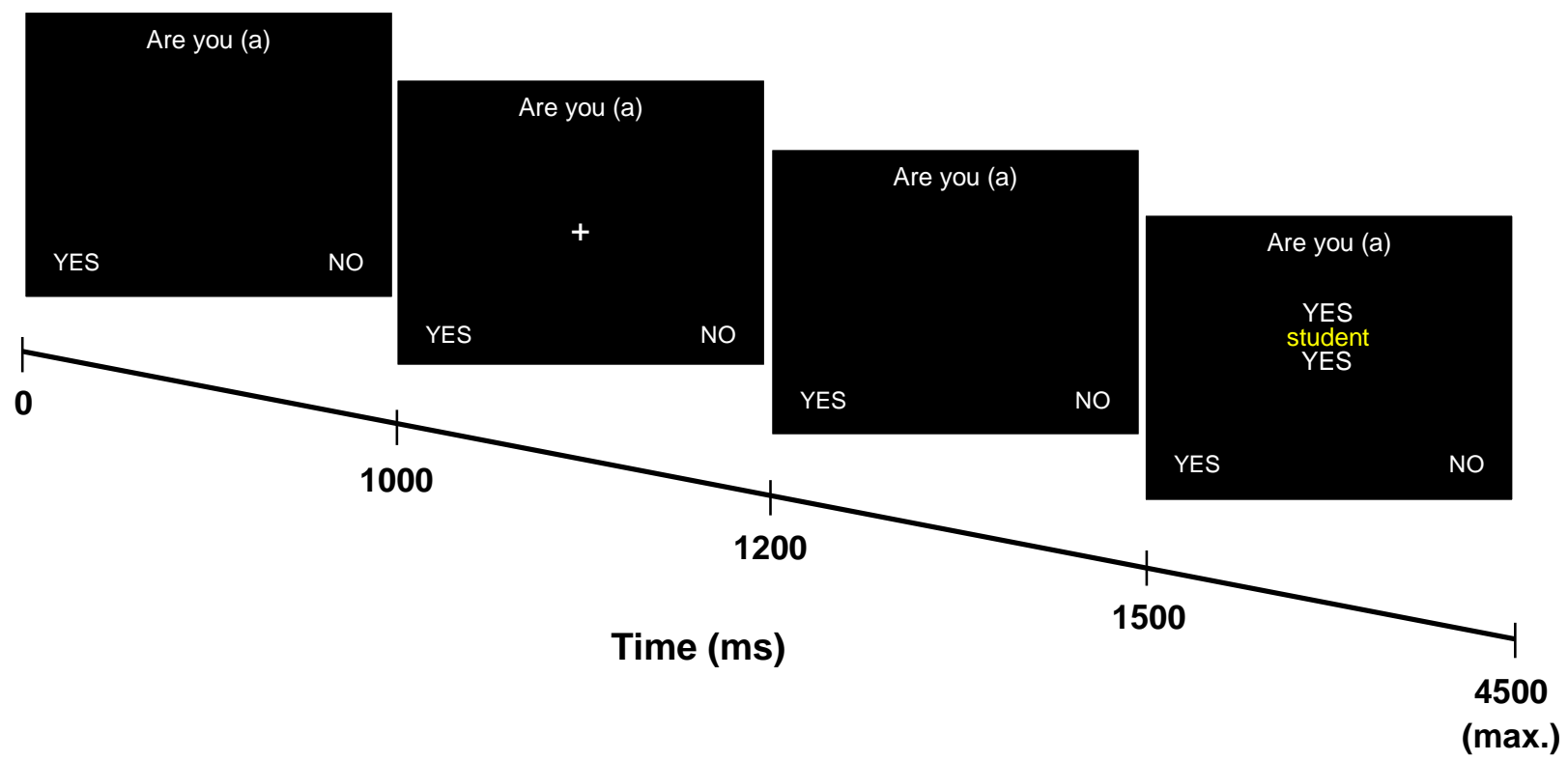

Figure 1. Flow of a trial in the Sheffield lie test.

\subsection{Results}

We present two result sections. In the first section, we focus on the crucial comparison of truth and lie distractor trials. Both error rates and reaction times were submitted to a Deception (Truth vs. Lie) x Distractor (Truth distractor vs. Lie distractor) repeated-measures analysis of variance (ANOVA). The second section focuses on analyses including neutral distractor trials. We added neutral distractor trials to our design for an exploratory purpose, that is, to see how facilitating or interfering the lie and truth distractors were compared to baseline. Accordingly, we compared neutral distractor trials with truth and lie distractor trials by means of Deception (Truth vs. Lie) x Distractor (Neutral distractor vs. Truth distractor vs. Lie distractor) ANOVAs on error rates and reaction times. We must note, however, that what 
is the most appropriate neutral baseline has been subject to debate in psycholinguistic research (Jonides \& Mack, 1984; Neely, 1991). Neutral trials may have unknown properties that render them dubious as baseline. Also with regard to our experiment, it seems reasonable to argue that the neutral and informative distractors may differ in the levels of alertness they evoke, and in their need for processing capacity. The lie and truth distractors may induce more relevance-orienting than the neutral distractors as they are linked to the task-relevant responses (Klein, 1964). Furthermore, the truth and lie distractors are meaningful words, which puts a higher load on linguistic and semantic processes than the meaningless string of neutral hatch signs (Klein, 1964). If neutral distractors were therefore processed more easily, facilitation effects may be underestimated and interference effects overestimated. The analyses should therefore be interpreted with caution.

Trials without response within the $3 \mathrm{~s}$ deadline $(1.37 \%)$ and trials with latencies below $300 \mathrm{~ms}(0.34 \%)$ were discarded. For reaction time analyses, we excluded trials with errors $(10.48 \%)$, and truth and lie trials with reaction times that were 2.5 standard deviations $(S D s)$ removed from each participant's mean reaction time on truth and lie trials, respectively $(2.08 \%)$. For ANOVAs, we calculated Cohen's effect size $f$ using the following formula: $f=$ $\sqrt{ }\left[\eta_{p}^{2} p /\left(1-\eta_{p}^{2}\right)\right]$. Cohen (1992) considered $f$ s of $0.10,0.25$, and 0.40 , to constitute small, medium, and large effects, respectively. For $t$-tests, we used Cohen's $d$ as an effect size, with $d$ values of $0.20,0.50$, and 0.80 respectively representing small, medium, and large effects (Cohen, 1988). Mean error rates and reaction times per trial type are presented in Table 1.

\subsubsection{Truth distractor trials versus lie distractor trials}

2.3.1.1. Errors. The analysis revealed main effects of Deception, $F(1,25)=35.19, p<$ $.001, f=1.19$, and Distractor, $F(1,25)=10.67, p<.01, f=0.65$. However, these effects were subsumed under a significant Deception by Distractor interaction, $F(1,25)=7.95, p<.01, f=$ 0.56. Follow-up $t$-tests indicated that there was a significant distractor effect on lie trials, 
showing that lying was more error-prone in the presence of lie distractors than in the presence of truth distractors, $t(25)=3.73, p<.01, d=0.73$. On truth trials, numerically, also truth telling was more error-prone with lie distractors than with truth distractors, but this difference was not significant, $t(25)=1.66, p=.11, d=0.32$. We also analyzed this interaction from the perspective of the lie effect, that is, the difference in error rates between lying and truth telling. From this perspective, the lie effect (i.e., Errors ${ }_{\mathrm{LIE}}$ minus $_{\text {Errors }} \mathrm{TRUTH}_{\mathrm{T}}$ ) was significant on both distractor type trials, $p \mathrm{~s}<.001$, but was larger on lie distractor trials than on truth distractor trials.

2.3.1.2. Reaction times. The main effect of Deception, $F(1,25)=93.52, p<.001, f=$ 1.94, and Distractor, $F(1,25)=30.02, p<.001, f=1.10$, were subsumed under a marginally significant Deception by Distractor interaction, $F(1,25)=4.19, p=.05, f=0.41$. The distractor effect was somewhat larger on trials on which participants told the truth, $t(25)=$ $5.05, p<.001, d=1.00$, than on which they lied, $t(25)=2.29, p=.03, d=0.45$. This resulted in a larger lie effect (i.e., $\mathrm{RT}_{\mathrm{LIE}}$ minus $\mathrm{RT}_{\mathrm{TRUTH}}$ ) on truth distractor trials than on lie distractor trials (though both were significantly different from zero, $p s<.001$ ).

\subsubsection{Truth and lie distractor trials versus neutral distractor trials}

2.3.2.1. Errors. The analysis revealed main effects of Deception, $F(1,25)=47.85, p<$ $.001, f=1.38$, and Distractor, $F(2,50)=7.46, p<.01, f=0.55$. However, these effects were subsumed under a significant Deception by Distractor interaction, $F(2,50)=3.63, p=.03, f=$ 0.38. Therefore, two follow-up ANOVAs were conducted, in which neutral distractor trials were compared to truth and lie distractor trials, separately. The follow-up ANOVA that compared neutral and truth distractor trials showed a significant main effect of Deception, $F(1,25)=41.06, p<.001, f=1.28$, and a trend toward a Deception by Distractor interaction, $F(1,25)=3.47, p=.07, f=0.37$. Follow-up $t$-tests revealed that only on lie trials a trend toward a difference between distractor types emerged, showing less errors on truth distractor 
trials than on neutral distractor trials, $t(25)=1.75, p=.09, d=0.34$. No difference was found on truth trials, $t<1$. The follow-up ANOVA that compared neutral and lie distractor trials yielded a main effect of Deception, $F(1,25)=42.59, p<.001, f=1.30$, as well as a main effect of Distractor, $F(1,25)=7.89, p=.01, f=0.56$, revealing more errors on lie distractor trials $(M=12.55 \%, S D=6.24)$ than on neutral distractor trials $(M=10.63 \%, S D=6.24)$. The Deception by Distractor interaction did not reach significance, $F<1$.

2.3.2.2. Reaction times. The ANOVA demonstrated a main effect of Deception, $F(1$, $25)=91.58, p<.001, f=1.92$. Also the main effect of Distractor proved significant, $F(2,50)$ $=27.81, p<.001, f=1.06$. Follow-up $t$-tests showed that response times on neutral distractor trials $(M=1168 \mathrm{~ms}, S D=218)$ were faster than on truth distractor trials $(M=1189 \mathrm{~ms}, S D=$ $230), t(25)=2.65, p=.01, d=0.51$, and lie distractor trials $(M=1225 \mathrm{~ms}, S D=239), t(25)=$ $6.50, p<.001, d=1.27$. The Deception by Distractor interaction remained non-significant, $F(2,50)=1.63, p=.21$.

Table 1

Mean error rates and reaction times in the Sheffield lie test of Experiment 1

\begin{tabular}{|c|c|c|c|c|}
\hline & $\begin{array}{l}\text { Neutral distractor } \\
\qquad M(S D)\end{array}$ & $\begin{array}{l}\text { Truth distractor (TD) } \\
\qquad M(S D)\end{array}$ & $\begin{array}{l}\text { Lie distractor (LD) } \\
\qquad M(S D)\end{array}$ & $\begin{array}{l}\text { Distractor effect } \\
\quad(\mathrm{LD}-\mathrm{TD}) \\
M(S D)\end{array}$ \\
\hline \multicolumn{5}{|l|}{ Error rates $(\%)$} \\
\hline Truth telling & $8.00(6.92)^{\mathrm{a}}$ & $8.12(6.95)^{a, b}$ & $9.55(6.18)^{b}$ & $1.43(4.41)$ \\
\hline Lying & $13.25(6.51)^{\mathrm{a}}$ & $11.19(7.55)^{\mathrm{a}}$ & $15.56(7.43)^{b}$ & $4.37(5.98)$ \\
\hline Lie effect (lie - truth) & $5.25(5.10)^{\mathrm{a}}$ & $3.07(3.72)^{\mathrm{a}, \mathrm{b}}$ & $6.01(5.55)^{\mathrm{a}, \mathrm{c}}$ & \\
\hline \multicolumn{5}{|l|}{ Reaction times (ms) } \\
\hline Truth telling & $1078(214)^{a}$ & $1094(224)^{b}$ & $1145(234)^{c}$ & $51(52)$ \\
\hline Lying & $1263(236)^{a}$ & $1289(247)^{b}$ & $1311(255)^{\mathrm{c}}$ & $22(48)$ \\
\hline Lie effect (lie - truth) & $185(121)^{\mathrm{a}}$ & $195(92)^{\mathrm{a}, \mathrm{b}}$ & $166(111)^{\mathrm{a}, \mathrm{c}}$ & \\
\hline
\end{tabular}

${ }_{\text {a,b,c }}$ Different letters in the same row indicate that the values are significantly different $(p<.05)$ 


\subsection{Discussion}

The aim of the present experiment was to test the hypothesis that constructing a lie involves a two-step process, in which the first step comprises the activation of the truth, so that based on the truth an alternative response can be generated in the second step. In a Sheffield lie test, participants lied or told the truth to yes/no questions that were flanked by distractors depicting either the truth (truth distractors) or lie response (lie distractors). Lying was found to be faster and more accurate in the presence of truth distractors compared to lie distractors. These distractor effects can be interpreted as "covert congruency" effects. The fact that lying was easier in the presence of truth distractors than in the presence of lie distractors supports the idea that lying involved a covert step that was influenced by the distractors. Our data are thus supportive for the existence of a two-step process in the act of lying, where the activation of the truth plays an essential role.

Although truth distractors facilitated lying relative to lie distractors, lying was slower on truth distractor trials than on neutral distractor trials. Hence, we cannot conclude that truth distractors facilitated lying relative to a neutral baseline. However, as discussed above, there are good reasons to believe that the distractors presented on the neutral distractor trials did not provide an adequate baseline. Most importantly, unlike the truth distractors, neutral distractors did not correspond to existing words and were not part of the set of potential responses (see Klein, 1964, for evidence showing that both factors influence reaction times).

Finally, a distractor effect was present also on trials that required a truth response. This distractor effect can be interpreted as an "overt congruency" effect: Whereas truth distractors are congruent with the to-be-emitted overt truth response, lie distractors are incongruent with that response. This finding confirms the idea that generating the truth is a single-step process, which merely requires the retrieval of the truth response. 


\section{Experiment 2}

The goal of our second experiment was twofold. First, we wanted to explore whether we could replicate the distractor effects found in Experiment 1. Second, we aimed to further validate our explanation for the effects in terms of (covert) response congruency. We did this by manipulating the proportion of truth distractor trials in the Sheffield lie test. We reasoned that if the distractor effects are due to response congruency - and thus operate at a response selection level - we would find similar proportion congruency effects as seen in typical response congruency tasks, such as the Stroop task. Stroop-like congruency effects increase as the proportion of congruent trials increases (e.g., Hommel, 1994; Logan \& Zbrodoff, 1979; Toth et al., 1995). To explain this phenomenon, it has mostly been argued that participants aggregate response-related information from the irrelevant and relevant dimension, and topdown modulate their attention to the irrelevant information depending on the proportion of congruent trials (Botvinick, Braver, Barch, Carter, \& Cohen, 2001; Logan \& Zbrodoff, 1979; but see Schmidt \& De Houwer, 2011). For instance, in a Stroop task with a relatively high proportion of congruent trials, the response associated with the task-irrelevant information (i.e., color words) is most often in line with the correct response. To optimize performance, participants could therefore strategically increase the attentional weight given to the words. This bias would facilitate responding on congruent trials, but heighten interference on incongruent trials. As such, relatively large congruency effects would be established. In contrast, a high proportion of incongruent trials could encourage participants to weigh irrelevant information to a lesser extent. This would decrease both facilitation on congruent trials and interference on incongruent trials, making for relatively small congruency effects.

In our experiment, participants performed a Sheffield lie test that either contained $25 \%$ or $75 \%$ truth distractor trials. From a covert response congruency account, we expected that participants in the $75 \%$ truth distractor group would assign more attentional weight to the 
distractors compared to the $25 \%$ truth distractor group. The $75 \%$ truth distractor group may strategically increase their attention toward the distractors because distractors most often represent the truth. Hence, in the $75 \%$ truth distractor condition, attending the distractors most often facilitates covert responding on lie trials (i.e., the initial activation of the truth) or overt responding on the truth trials. On the other hand, the $25 \%$ group may shift their attention away from the distractors because distractors represent the lie, which complicates covert responding on lie trials and overt responding on truth trials. Hence, we expected to find larger distractor effects in the $75 \%$ truth distractor group than in the $25 \%$ truth distractor group.

\subsection{Method}

3.1.1. Participants. Fifty-five undergraduate students at Ghent University (11 men, $M_{\mathrm{age}}=19.04$ years, $\left.S D=2.47\right)$ took part in the experiment. In return for their participation, they received course credits. All participants had normal or corrected-to-normal vision.

3.1.2. Apparatus. The Sheffield lie test was presented on a Pentium IV personal computer with a 17-inch color monitor running Tscope (Stevens et al., 2006). Participants were seated approximately 50 centimeters from the screen.

\subsection{Procedure}

Participants were tested in a dimly lit room and were randomly assigned to either the $25 \%$ truth distractor group $(n=28)$ or the $75 \%$ truth distractor group $(n=27)$. As in Experiment 1, participants first filled out a questionnaire consisting of the questions that would be presented in the lie test. Response options were again yes, no or unsure. For design purposes, we now used 24 questions (see Appendix B). Half of them could be answered truthfully by a "yes" answer, and the other half by a "no" answer. When participants indicated they were unsure $(<1 \%)$, or gave an unexpected answer $(<1 \%)$, we removed their behavioral responses to these particular questions in the Sheffield lie test (1.83\% data loss). 
Participants first performed 12 practice trials without distractors, followed by 8 practice trials with lie or truth distractors. Next, 384 test trials were presented. In contrast with Experiment 1, neutral distractor trials were no longer displayed. In the $25 \%$ truth distractor group, truth distractors were presented on $25 \%$ of the trials, and lie distractors on the remaining $75 \%$ of the trials. Conversely, in the $75 \%$ truth distractor group, the distractors represented the truth response on $75 \%$ of the trials, and the lie response on $25 \%$ of the trials. Other task characteristics were the same as in Experiment 1.

\subsection{Results}

Trials without response within the $3 \mathrm{~s}$ deadline (5.37\%) and response latencies below $300 \mathrm{~ms}(0.18 \%)$ were discarded. Six participants were excluded from the analysis: (1) two participants because they used the wrong response keys during the task, (2) three participants because of a programming error, and (3) one participant who had an exceedingly high proportion of trials without response $(29.69 \%)$. Eventually, 24 participants remained in the $25 \%$ truth distractor group and 25 participants in the $75 \%$ truth distractor group. Groups did not differ for age, $t<1$, or gender, $\chi^{2}(1, N=49)=1.08, p=.30$. On both error rates and reaction times, we ran a repeated measures ANOVA with Deception (Truth vs. Lie) and Distractor (Truth distractor vs. Lie distractor) as within-subjects variables and Group (25\% truth distractor group vs. $75 \%$ truth distractor group) as a between-subjects variable. Mean error rates an reaction times per trial type are presented in Table 2.

3.3.1. Errors. The analysis revealed significant main effects of Deception, $F(1,47)=$ 56.64, $p<.001, f=1.10$, and Distractor, $F(1,47)=53.44, p<.001, f=1.07$. However, there was also a marginally significant Deception by Distractor interaction, $F(1,47)=3.60, p=.06$, $f=0.28$. A follow-up analysis showed that both on lie and truth trials the distractor effect was significant, $p s<.001$, but the effect tended to be larger on lie trials. As a result, the lie effect was larger on lie distractor trials than on truth distractor trials, yet both were significant, $p \mathrm{~s}<$ 
.001. The crucial Distractor by Group interaction also proved significant, $F(1,47)=4.91, p=$ $.03, f=0.32$, showing that the distractor effects were larger in the $75 \%$ truth distractor group than in the $25 \%$ truth distractor group (yet all were significantly different from zero, $p \mathrm{~s} \leq$ .02). Other main and interaction effects were not significant, $F \mathrm{~s}<1$.

3.3.2. Reaction times. For the analysis of reaction times, we removed trials with incorrect responses $(10.89 \%)$, and truth and lie trials with reaction times that were more than 2.5 SDs removed from each participant's mean reaction time on truth and lie trials, respectively $(1.64 \%)$. The main effect of Deception, $F(1,47)=262.52, p<.001, f=2.36$, revealed a significant lie effect in reaction times. The main effect of Distractor, $F(1,47)=$ 23.73, $p<.001, f=0.71$, was subsumed under a significant Distractor by Group interaction, $F(1,47)=7.71, p<.01, f=0.41$. Like in the errors, the distractor effects in reaction times were larger in the $75 \%$ truth distractor group than in the $25 \%$ truth distractor group. Followup $t$-tests are in line with this finding: The distractor effect was significantly different between groups on lie trials, $t(47)=2.46, p=.02, d=0.70$, and followed a trend on truth trials, $t(47)=$ $1.74, p=.09, d=0.50$. The distractor effects were not significant in the $25 \%$ truth distractor group, $t \mathrm{~s} \leq 1.54$ (truth telling: $d=0.31$; lying: $d=0.14$ ), whereas they were highly significant in the $75 \%$ truth distractor group, $p$ s $\leq .001$ (truth telling: $d=0.90$; lying: $d=0.80$ ). Other main and interaction effects did not reach significance, $F_{\mathrm{s}}<1$.

\subsection{Discussion}

Experiment 2 aimed to further strengthen the idea that lie construction can involve a two-step process by (1) replicating the distractor effects found in Experiment 1, and (2) validating the explanation that these distractor effects stem from covert response congruency. Participants performed a Sheffield lie test that either contained $25 \%$ or $75 \%$ truth distractor trials. From the hypothesis that the distractor effects on lie trials can be considered as "covert response congruency" effects, we expected to find proportion congruency effects as found in 
typical response congruency tasks, such as the Stroop task. Our results indeed supported this hypothesis in both errors and reaction times: The distractor effects on lie trials were significantly larger in the $75 \%$ truth distractor group compared to the $25 \%$ truth distractor group. The reaction-time distractor effects even tripled in effect size. The presence of Strooplike proportion congruency effects speaks for the idea that covert response congruency underlies the distractor effects found on lie trials. As such, our findings confirm the hypothesis that constructing lies can require a first step of truth activation.

\section{General Discussion}

Previous research holds that the higher cognitive demand of lying compared to truth telling is partly due to the initial activation of the dominant truth response (e.g., Christ et al., 2009; Duran, Dale, \& McNamara, 2010). Cognitive control would be needed to solve the response conflict that arises between the activated truth and the to-be-emitted lie response. However, we reasoned that the truth may also serve the lying process as it may be involved in constructing the lie. In line with this idea, self-reports have revealed that liars frequently alter the truth or simply say the opposite of what is true (Walczyk et al., 2003). These reports suggest that constructing a lie may occur via a two-step process, where the first step entails the activation of the truth, based upon which a deceptive response can be formulated in a second step. Because the accuracy of introspective self-reports on cognition can be questioned (e.g., Nisbett \& Wilson, 1977), our study intended a more direct test of the two-step hypothesis of lying by applying an experimental manipulation in the Sheffield lie test, a reaction-time based deception test in which participants alternatingly lie and tell the truth to yes/no questions. Inspired by Seymour's 1977 paper, we flanked the questions by distractors that either represented the truth or lie response.

Experiment 1 showed that lying was faster and more accurate when the questions were flanked by truth distractors compared to lie distractors. In line with the two-step hypothesis of 


\section{Table 2}

Mean error rates and reaction times in the Sheffield lie test of Experiment 2

\begin{tabular}{|c|c|c|c|c|c|c|}
\hline & \multicolumn{3}{|c|}{$25 \%$ truth distractor group } & \multicolumn{3}{|c|}{$75 \%$ truth distractor group } \\
\hline & $\begin{array}{l}\text { Truth distractor (TD) } \\
\qquad M(S D)\end{array}$ & $\begin{array}{c}\text { Lie distractor (LD) } \\
M(S D)\end{array}$ & $\begin{array}{c}\text { Distractor effect } \\
(\mathrm{LD}-\mathrm{TD}) \\
M(S D)\end{array}$ & $\begin{array}{l}\text { Truth distractor (TD) } \\
\qquad M(S D)\end{array}$ & $\begin{array}{c}\text { Lie distractor (LD) } \\
M(S D)\end{array}$ & $\begin{array}{c}\text { Distractor effect } \\
(\mathrm{LD}-\mathrm{TD}) \\
M(S D)\end{array}$ \\
\hline \multicolumn{7}{|l|}{ Error rates $(\%)$} \\
\hline Truth telling & $6.45(4.65)$ & $8.91(5.45)$ & $2.46(4.68)^{\mathrm{a}}$ & $6.12(4.42)$ & $10.38(7.50)$ & $4.26(4.51)^{\mathrm{b}}$ \\
\hline Lying & $11.38(6.83)$ & $14.89(7.59)$ & $3.51(4.79)^{\mathrm{a}}$ & $10.57(7.77)$ & $17.47(8.71)$ & $6.90(6.93)^{b}$ \\
\hline Lie effect (lie - truth) & $4.93(6.53)$ & $5.98(6.26)$ & & $4.45(5.51)$ & $7.09(6.59)$ & \\
\hline \multicolumn{7}{|l|}{ Reaction times (ms) } \\
\hline Truth telling & $1254(230)$ & $1280(204)$ & $26(83)^{\mathrm{a}}$ & $1200(156)$ & $1265(183)$ & $65(72)^{\mathrm{b}}$ \\
\hline Lying & $1480(247)$ & $1491(242)$ & $11(80)^{\mathrm{a}}$ & $1437(193)$ & $1508(203)$ & $71(89)^{\mathrm{b}}$ \\
\hline Lie effect (lie - truth) & $226(141)$ & $211(92)$ & & $237(100)$ & $243(111)$ & \\
\hline
\end{tabular}

${ }^{\mathrm{a}, \mathrm{b}}$ Different letters in the same row indicate that the values are significantly different $(p<.05)$ 
lie construction, these distractor effects can be interpreted as "covert congruency" effects. Truth distractors would be congruent and lie distractors incongruent with the covert truth response that is activated in the first step. Relative to lie distractors, truth distractors would thus facilitate the truth activation and help forward the construction of the lie in the second step. To reinforce the idea that the distractor effects can be explained by covert response congruency, Experiment 2 manipulated the proportion of truth distractors. We predicted that a high proportion of truth distractor trials would stimulate participants to strategically increase their attention toward the distractors and/or a high proportion of lie distractor trials would encourage participants to shift their attention away from the distractors. More attention for distractors may facilitate the first step of the two-step process on lie trials with truth distractors and/or amplify interference on this first step on lie trials with lie distractors, thereby establishing relatively large distractor effects. In keeping with this hypothesis, we found that the group who performed a Sheffield lie test that consisted of $75 \%$ truth distractor trials exhibited larger distractor effects on lie trials than the group who executed the test with only $25 \%$ truth distractor trials.

In line with previous research, our study supports the idea that the truth is activated during lying. However, in contrast to most previous studies that implied from this finding that the truth hampers lying (e.g., Spence et al., 2004; Johnson, Barnhardt, \& Zhu, 2004), our findings demonstrate that the truth may as well be functional in lying in that it can constitute an integral element of the construction of the lie. As such, our study adds to previous selfreport findings (Walczyk et al., 2003) by providing more direct support for the idea that the truth can be useful in the lying process. Our findings validate the notions of the ActivationDecision-Construction-Action Theory (Walczyk et al., 2014) and Working Model of Deception (Vendemia, Buzan, \& Simon-Dack, 2005) that point to the truth's usefulness in the act of lying. However, whereas these models advance the helpful nature of the truth in a rather 
non-explicit manner, and in the shadow of the conflict hypothesis, our study explicitly put forward the idea that the truth forms a first step in the construction of lies. The two-step hypothesis also clarifies the role of working memory updating and shifting in lying, as it gives a rationale why constructing a lie would require to maintain both the truth and lie response in working memory, and to shift between the mental sets associated with these responses.

We note that we also found distractor effects on trials on which participants told the truth. These effects showed that truth telling was more easy in the presence of truth distractors compared to lie distractors. This finding conforms to the idea that generating the truth only entails a single step, which is the mere activation of the truth response. Truth distractors are then congruent and lie distractors incongruent with the overt truth response. The fact that generating the truth would only entail one step, whereas constructing a lie would involve two steps, suggests that the cognitive cost of lying may be partly attributed to the extra step.

Our findings call for a shift in theoretical thinking on deception that not only highlights the interfering, but also the functional features of the truth activation in the act of lying. In that respect, one may wonder how our proposed two-step process could be modeled within a dynamical framework in which the truth and lie response compete. First, our findings suggest that the selection of the truth in Step 1 is influenced by the automatic processing of distractors. In other words, the decision at Step 1 could automatically be facilitated by truth distractors and/or inhibited by lie distractors. Given that cognitive and motor stages continuously blend into each other (see Song \& Nakayama, 2009, for an overview of dynamical models on decision making), effects of the covert truth activation and distractors would leak into this final motor output. From a dynamical perspective, we do not exclude that the temporal window of the two stages may overlap to some extent such that processes operating at Step 1 might feed forward to Step 2 even before Step 1 has been finalized. However, if we observe that truth distractors facilitate the selection of lie responses relative to 
lie distractors, this would strongly suggest that the selection of the lie response involves an initial step of truth retrieval.

Second, based on dynamical models on decision making (Song \& Nakayama, 2009), every step of response selection within the two-step process may involve a parallel competition between response options, in our case the response options being the truth and lie response. Because the lie would not yet be formulated in the first step of our proposed lying process, the lie response option may here represent the goal to execute a lie response. We assume that after the lie response has been constructed in the second step and needs to be executed, the truth response would no longer be functional and give rise to actual response conflict with the to-be-emitted lie. One important issue that future research should elucidate is the relative impact of the functional and interfering effect of the truth activation on lying proficiency. It is possible that the conflicting aspect of the truth activation impacts more heavily on lying proficiency than its functional facet. This would imply that lying in the presence of truth distractors would be less efficient (though still more efficient than in the presence of lie distractors) compared to lying in an appropriate baseline condition. A few findings are in line with the idea that the conflict-inducing effect of the truth activation would overpower its functional effect. Walcyzk and colleagues (2005), for example, found that being better able to access the truthful memory of an episodic event due to its recency increased the reaction-time lie effect compared to lying about less recent events. Furthermore, Farrow and co-workers showed that participants with better verbal working memory updating skills displayed larger reaction-time lie effects as a result of faster truth telling, but slower deceptive responding compared to participants with less efficient updating skills (Farrow, Hopwood, Parks, Hunter, \& Spence, 2010). The authors reasoned that those with better updating skills retrieved more strongly the truth, which caused more response conflict and complicated its inhibition during deception. 
To get a better picture of the temporal characteristics of the two-step process involved in lying, future research may manipulate the stimulus onset asynchrony (SOA) between the question and the distractor. One could expect that with increasing SOAs, distractors would no longer affect the first "truth" step, but interact with the second "lie" step. As a result, "overt congruency" effects would be established. Manipulating the SOA could reveal when the "covert congruency" effects turn into "overt congruency" effects, and as such give an indication at which point the truth loses its functional character and starts to interfere.

It is important to realize that we investigated the two-step hypothesis of lie construction in the specific context where participants were instructed to lie to fairly untrained yes/no questions. One should therefore be careful in generalizing our findings to lying in other situations. As self-reports have revealed, lying to yes/no questions often happens by applying the simple rule "say the opposite of what is true" (Walczyk et al., 2003). In that case, the lie response is highly related to the truth. Future research should investigate whether a two-step process of lie construction also applies to open-ended questions, where the link between the truth and the lie is often less obvious. Self-reports do show that the truth is also used when lying to open-ended questions (Walczyk et al., 2003), but additional experimental research on this issue seems warranted. Although our participants lied several times to the same questions, we cannot speak of extended lie training (e.g., Van Bockstaele et al., 2012; Verschuere et al., 2011). It is possible that lying does no longer involve a two-step process when a lie has been extensively practiced. The strong association between the question and the lie response may then lead to the immediate retrieval of the lie, without the requirement of the intermediate "truth" step. When this hypothesis proves true, one could expect to find "overt congruency" effects, where lie distractors would be more helpful than truth distractors when lying.

Our study investigated lying from the definition that all forms of deception eventually require a response that is incompatible with the truth (Johnson, Barnhardt, \& Zhu, 2004). We 
acknowledge, though, that our questions are not the kind of questions people usually lie to in real life. However, it was a well-considered choice to examine the effect of distractors in a restricted design using these simple, fairly neutral questions. From this approach, we were able to exclude possible effects of factors that may be at play when people lie in real life, such as emotions or motivation. Having found the "basic" distractor effect, we can now systematically investigate the effects of these factors in more real-life settings.

Our research contributes not only to the theoretical understanding of the mechanisms underlying deception, but also provides a possible means to detect deception. In an interrogation context, a suspect could be presented with a "Did you do it?"-like question. The examinee is expected to provide an overt "no" answer - whether guilty or not (as an affirmative response implies confessing crime enactment). Interestingly, this overt "no" answer involves the truth for innocents, but a lie for guilty suspects. One could be tempted to infer deception simply from the response latencies to the did-you-do-it question. However, such inference would necessarily be based upon a between-subjects comparison, that (1) requires a database of how fast innocents respond, and even more problematic (2) is biased by individual differences in general response speed. The use of distractors circumvents these problems, as it allows to infer deception from a within-subjects comparison of how truth versus lie distractors influence performance. When denying the did-you-do-it question, our findings suggests that "yes" and "no" distractors will differentially impact upon the overt "no" response in guilty versus innocent examinees. For guilty suspects, "yes" distractors (the covert truth) should facilitate responding compared to "no" distractors. The opposite pattern is expected in innocent suspects, where the "no" distractors (the covert truth) should facilitate responding compared to "yes" distractors. Therefore, rather than the response to the question per se, the manner in which performance to the question is influenced by "yes" and "no" distractors, could bring guilt to light. 


\section{References}

Abe, N. (2009). The neurobiology of deception: evidence from neuroimaging and loss-offunction studies. Current Opinion in Neurology, 22, 594-600. doi:10.1097/WCO.0b013e328332c3cf

Abe, N. (2011). How the brain shapes deception: An integrated review of the literature. Neuroscientist, 17, 560-574. doi:10.1177/1073858410393359

Botvinick, M. M., Braver, T. S., Barch, D. M., Carter, C. S., \& Cohen, J. D. (2001). Conflict monitoring and cognitive control. Psychological Review, 108, 624-652. doi:10.1037/0033-295X.108.3.624

Christ, S. E., Essen, D. C., Watson, J. M., Brubaker, L. E., \& McDermott, K. B. (2009). The contributions of prefrontal cortex and executive control to deception: Evidence from activation likelihood estimate meta-analyses. Cerebral Cortex, 19, 1557-1566. doi:10.1093/cercor/bhn189

Clark, H. H., \& Chase, W. G. (1972). On the process of comparing sentences against pictures. Cognitive Psychology, 3, 472-517. doi: 10.1016/0010-0285(72)90019-9

Cohen, J. (1988). Statistical power analysis for the behavioural sciences. Hillsdale, NJ: Lawrence Erlbaum.

Cohen, J. (1992). A power primer. Psychological Bulletin, 112, 155-159. doi:10.1037/0033-2909.112.1.155

Duran, N. D., Dale, R., \& McNamara, D. S. (2010). The action dynamics of overcoming the truth. Psychonomic Bulletin and Review, 17, 486-491. doi:10.3758/PBR.17.4.486

Evans, A. D., \& Lee, K. (2013). Emergence of lying in very young children. Developmental Psychology, 49, 1958-1963. doi:10.1037/a0031409 
Farrow, T. F., Hopwood, M. C., Parks, R. W., Hunter, M. D., \& Spence, S. A. (2010). Evidence of mnemonic ability selectively affecting truthful and deceptive response dynamics. American Journal of Psychology, 123, 447-453. doi: 10.5406/amerjpsyc. 124.4 .0447

Gilbert, D. T. (1991). How mental systems believe. American Psychologist, 46, 107-119. doi: 10.1037/0003-066x.46.2.107

Gilbert, D. T., Krull, D. S., \& Malone, P . S. (1990). Unbelieving the unbelievable: Some problems in the rejection of false information. Journal of Personality and Social Psychology, 59, 601-613. doi: 10.1037/0022-3514.59.4.601

Hadar, A. A., Makris, S., \& Yarrow, K. (2012). The truth-telling motor cortex: Response competition in M1 discloses deceptive behaviour. Biological Psychology, 89, 495502. doi:10.1016/j.biopsycho.2011.12.019

Hala, S., \& Russell, J. (2001). Executive control within strategic deception: A window on early cognitive development? Journal of Experimental Child Psychology, 80, 112141. doi:10.1006/jecp.2000.2627

Hershkowitz, I. (2001). A case study of child sexual false allegation. Child Abuse and Neglect, 25, 1397-1411. doi:10.1016/S0145-2134(01)00274-5

Hommel, B. (1994). Spontaneous decay of response-code activation. Psychological Research, 56, 261-268. doi:10.1007/BF00419656

Johnson, R., Jr., Barnhardt, J., \& Zhu, J. (2004). The contribution of executive processes to deceptive responding. Neuropsychologia, 42, 878-901. doi:10.1016/j.neuropsychologia.2003.12.005

Jonides, J., \& Mack, R. (1984). On the cost and benefit of cost and benefit. Psychological Bulletin, 96, 29-44. doi:10.1037/0033-2909.96.1.29

Klein, G. S. (1964). Semantic power measured through the interference of words with color- 
naming. American Journal of Psychology, 77, 576-588. doi:10.2307/1420768

Kornblum, S., Hasbroucq, T., \& Osman, A. (1990). Dimensional overlap: Cognitive basis for stimulus-response compatibility-A model and taxonomy. Psychological Review, 97, 253-270. doi:10.1037/0033-295X.97.2.253

Logan, G. D., \& Zbrodoff, N. J. (1979). When it helps to be misled: Facilitative effects of increasing the frequency of conflicting stimuli in a Stroop-like task. Memory \& Cognition, 7, 166-174. doi:10.3758/s13414-011-0261-0

Lykken, D. T. (1959). The GSR in the detection of guilt. Journal of Applied Psychology, 43, 385-388. doi:10.1037/h0046060

MacLeod, C. M. (1991). Half a century of research on the Stroop effect: An integrative review. Psychological Review, 109, 163-203. doi:10.1037/0033-2909.109.2.163

Neely, J. H. (1991). Semantic priming effects in visual word recognition: A selective review of current findings and theories. In D. Besner \& G. W. Humphreys (Eds.), Basic processes in reading: Visual word recognition (pp. 264-336). Hillsdale, NJ: Erlbaum.

Nisbett, R. E., \& Wilson, T. D. (1977). Telling more than we can know: Verbal reports on mental processes. Psychological Review, 84, 231-259. doi:10.1037/0033-295X.84.3.231

Pronin, E. (2009). The Introspection Illusion. In M. P. Zanna (Ed.). Advances in Experimental Social Psychology (Vol. 41, pp. 1-67). New York, NY: Academic Press. doi:10.1016/S0065-2601(08)00401-2

Schmidt, J. R., \& De Houwer, J. (2011). Now you see it, now you don't: Controlling for contingencies and stimulus repetitions eliminates the Gratton effect. Acta Psychologica, 138, 176-186. doi:10.1016/j.actpsy.2011.06.002

Seymour, P. H. K. (1977). Conceptual encoding and locus of the Stroop effect. Quarterly Journal of Experimental Psychology, 29, 245-265. doi:10.1080/14640747708400601 
Seymour, T. L. (2001). A EPIC model of the "guilty knowledge effect": Strategic and automatic processes in recognition. Dissertation Abstracts International: Section B: The Sciences and Engineering, 61, 5591.

Seymour, T. L., \& Schumacher, E. H. (2009). Electromyographic evidence for response conflict in the exclude recognition task. Cognitive, Affective, \& Behavioral Neuroscience, 9, 71-82. doi:10.3758/CABN.9.1.71.

Song, J.-H., \& Nakayama, K. (2009). Hidden cognitive states revealed in choice reaching tasks. Trends in Cognitive Sciences, 13, 360-366. doi:10.1016/j.tics.2009.04.009

Spence, S. A., Farrow, T. F. D., Herford, A. E., Wilkinson, I. D., Zheng, Y., \& Woodruff, P. W. R. (2001). Behavioural and functional anatomical correlates of deception in humans. NeuroReport, 12, 2849-2853. doi:10.1097/00001756-200109170-00019

Spence, S. A., Hunter, M. D., Farrow, T. F. D., Green, R. D., Leung, D. H., Hughes, C. J., \& Ganesan, V. (2004). A cognitive neurobiological account of deception: evidence from functional neuroimaging. Philosophical Transactions of the Royal Society B: Biological Sciences, 359, 1755-1762. doi:10.1098/rstb.2004.1555

Spence, S. A., Kaylor-Hughes, C., Farrow, T. F., \& Wilkinson, I. D. (2008). Speaking of secrets and lies: the contribution of ventrolateral prefrontal cortex to vocal deception. Neuroimage, 40, 1411-1418. doi:10.1016/j.neuroimage.2008.01.035

Spinoza, B. (1982). The ethics and selected letters. (S. Feldman, Ed., and S. Shirley, Trans.). Indianapolis, IN: Hackett. (Original work published 1677)

Stevens, M., Lammertyn, J., Verbruggen F., \& Vandierendonck A. (2006). Tscope: A C library for programming cognitive experiments on the MS Windows platform. Behavior Research Methods, 38, 280-286. doi:10.3758/BF03192779

Talwar, V., \& Lee, K. (2008). Socio-cognitive correlates of children's lying behavior. Child Development, 79, 866-881. doi:10.1111/j.1467-8624.2008.01164.x 
Toth, J. P., Levine, B., Stuss, D. T., Oh, A., Winocur, G., \& Meiran, N. (1995). Dissociation of processes underlying S-R compatibility: Evidence for the independent influence of what and where. Consciousness \& Cognition, 4, 483-501. doi:10.1006/cog.1995.1052

Van Bockstaele, B., Verschuere, B., Moens, T., Suchotzki, K., Debey, E., \& Spruyt, A. (2012). Learning to lie: effects of practice on the cognitive cost of lying. Frontiers in Psychology, 3, 526. doi:10.3389/fpsyg.2012.00526

Vendemia, J. M. C., Buzan, R. F., \& Simon-Dack, S. L. (2005). Reaction time of motor responses in two-stimulus paradigms involving deception and congruity with varying levels of difficulty. Behavioural Neurology, 16, 25-36. doi: 10.1155/2005/804026

Vendemia, J. M. C., Schillaci, M. J., Buzan, R. F., Green, E. P., \& Meek, S. W. (2009). Alternate technologies for the detection of deception. In D. T. Wilcox (Ed.), The use of the polygraph in assessing, treating and supervising sex offenders (pp. 267-296). West Sussex, UK: Wiley-Blackwell.

Verschuere, B., Ben-Shakhar, G., \& Meijer, E. (2011). Memory detection: Theory and application of the Concealed Information Test. Cambridge, UK: Cambridge University Press.

Verschuere, B., \& De Houwer, J. (2011). Detecting concealed information in less than a second: Response-latency based measures. In B. Verschuere, G. Ben-Shakhar, \& E. Meijer (Eds.), Memory detection. London, UK: Cambridge University Press. doi:10.1017/CBO9780511975196.004

Verschuere, B., Spruyt, A., Meijer, E. H., \& Otgaar, H. (2011). The ease of lying. Consciousness and Cognition, 20, 908-911. doi: 10.1016/j.concog.2010.10.023

Visu-Petra, G., Varga, M., Miclea, M., \& Visu-Petra, L. (2013). When interference helps: increasing executive load to facilitate deception detection in the concealed information 
test. Frontiers in Psychology, 4, 146. doi: 10.3389/fpsyg.2013.00146

Vrij A., Fisher R. E., Mann S., \& Leal S. (2006). Detecting deception by manipulating cognitive load. Trends in Cognitive Sciences, 10, 141-142. doi:10.1016/j.tics.2006.02.001

Vrij, A., Granhag, P. A., \& Mann, S. (2010). Good liars. The Journal of Psychiatry and Law, 38, 77-98.

Walczyk, J. J., Harris, L. L., Duck, T. K., \& Mulay, D. (2014). A social-cognitive framework for understanding serious lies: Activation-Decision-Construction-Action Theory. New Ideas in Psychology, 34, 22-36. doi: 10.1016/j.newideapsych.2014. 03.0

Walczyk, J. J., Mahoney, K. T., Doverspike, D., \& Griffith-Ross, D. A. (2009). Cognitive lie detection: Response time and consistency of answers as cues to deception. Journal of Business and Psychology, 24, 33-49. doi:10.1007/s10869-009-909

Walczyk, J. J., Roper, K. S., Seemann, E., \& Humphrey, A. M. (2003). Cognitive mechanisms underlying lying to questions: Response time as a cue to deception. Applied Cognitive Psychology, 17, 755-774. doi:10.1002/acp.914

Walczyk, J. J., Schwartz, J. P., Clifton, R., Adams, B., Wei, M., \& Zha, P. (2005). Lying person-to-person about life events: A cognitive framework for lie detection. Personnel Psychology, 58, 141-170. doi: 10.1111/j.1744-6570.2005.00484.x

Wilson, T. D. (2002). Strangers to ourselves: discovering the adaptive unconscious. Cambridge, MA: Belknap Press of Harvard University Press.

Zuckerman, M., DePaulo, B. M., \& Rosenthal, R. (1981). Verbal and nonverbal communication of deception. In L. Berkowitz (Ed.), Advances in Experimental Social Psychology (Vol. 14, pp. 1-57). New York, NY: Academic Press. doi: 10.1016/S0065-2601(08)60369-X 


\section{Footnotes}

${ }^{1}$ Our two-step hypothesis should be distinguished from the concept of "truth bias" (Gilbert, 1991; Spinoza, 1677/1982). Truth bias is the phenomenon that people have the tendency to verify incoming information as true. An extra step would then be required to falsify this initial belief if it is not in line with the actual truth. The idea of "truth bias" has been supported in studies using verification tasks, by showing that participants are faster to verify that a proposition is true than to verify it is false (Clark \& Chase, 1972; Gilbert, Krull, Malone, 1990). Whereas the concept of truth bias refers to how information is understood by the receiver (i.e., initial default assumption is that information is true), the two-step hypothesis of lying deals with how deceptive information is conveyed by the sender (i.e., first determine the truth in order to afterwards communicate the lie). 
Appendix A

Questions used in Experiment 1

\begin{tabular}{cl}
\hline $\begin{array}{c}\text { Questions that required a truthful } \\
\text { "yes" response* }\end{array}$ & $\begin{array}{c}\text { Questions that required a truthful } \\
\text { "no" response }\end{array}$ \\
\hline Are you a student? & \\
Are you a human? & Are you a professor? \\
Are you inside? & Are you an animal? \\
Are you a man? & Are you outside? \\
Are you a participant? & Are you a woman? \\
Are you a person? & Are you an experimenter? \\
Are you alive? & Are you an object? \\
Are you a boy? & Are you dead? \\
Are you a citizen? & Are you a girl? \\
Are you an adult? & Are you a soldier? \\
& Are you a toddler? \\
\hline
\end{tabular}

Note: *For female participants, the questions including the target words "man", "woman", "boy", and "girl" required the opposite response as indicated in the table. 
Appendix B

Questions used in Experiment 2

\begin{tabular}{cl}
\hline $\begin{array}{c}\text { Questions that required a truthful } \\
\text { "yes" response* }\end{array}$ & $\begin{array}{c}\text { Questions that required a truthful } \\
\text { "no" response }\end{array}$ \\
\hline Are you a student? & Are you a professor? \\
Are you a human? & Are you an animal? \\
Are you inside? & Are you outside? \\
Are you a man? & Are you a woman? \\
Are you a participant? & Are you an experimenter? \\
Are you a person? & Are you an object? \\
Are you alive? & Are you dead? \\
Are you a son? & Are you a daughter? \\
Are you a citizen? & Are you a soldier? \\
Are you an adult? & Are you a toddler? \\
Are you European? & Are you Asian? \\
Are you white? & Are you black? \\
& \\
\hline
\end{tabular}

Note: *For female participants, the questions including the target words "man" and "woman" required the opposite response as indicated in the table. 